\title{
Acute Administration of $d$-Amphetamine Decreases Impulsivity in Healthy Volunteers
}

\author{
Harriet de Wit, Ph.D., Justin L. Enggasser, M.A., and Jerry B. Richards, Ph.D.
}

This study investigated the acute behavioral effects of $d$-amphetamine on several behavioral indices of impulsivity. Impulsivity has been defined, variously, as difficulty in inhibiting inappropriate behaviors, inability to wait, insensitivity to delayed consequences or an alteration in the perception of time; standardized procedures have been developed to measure these behavioral dimensions. However, it is not known how drugs affect these measures, and few studies have examined more than one measure in a single study. In this study, 36 healthy men and women participated in three sessions, in which they received placebo, $10 \mathrm{mg}$, or 20 $m g d$-amphetamine in randomized order. On each session they performed the following five tasks: the Stop Task, which measures behavioral inhibition, a delay discounting task, which measures the relative value of immediate vs. delayed rewards, a delay of gratification task, a Go/No-Go task, and a time estimation task. Subjects also completed mood questionnaires. Amphetamine produced its expected subjective, mood-altering effects, including increases in POMS Friendliness and Elation scales, and ARCI Euphoria and Stimulant scales. On the measures of impulsivity, amphetamine decreased impulsive responding on three of the tasks: on the Stop Task it decreased Stop reaction times without affecting Go reaction time, on the Go/No-Go task, it decreased the number of false alarms, and on the delay discounting measure, amphetamine (20 mg) decreased $k$ values indicating less discounting of delayed reward. Other measures of impulsive behavior were unaffected. These results suggest that acute doses of amphetamine decrease several forms of impulsive behavior. These findings extend and confirm previous findings in humans and laboratory animals.

[Neuropsychopharmacology 27: 813-825, 2002] (C) 2002 American College of Neuropsychopharmacology. Published by Elsevier Science Inc.
KEY WORDS: $d$-Amphetamine; Impulsivity; Behavior; Subjective Effect; Human

Drug abuse is associated with a range of maladaptive behaviors that are described loosely as "impulsive."

From the Department of Psychiatry, The University of Chicago, Chicago, IL (HdW, JLE), and Behavioral Medicine, University of New York at Buffalo, Buffalo, NY (JBR).

Address correspondence to: H. de Wit, Department of Psychiatry, MC3077, The University of Chicago, 5841 S. Maryland Avenue, Chicago, IL 60637. Tel.: (773) 702-1537; Fax: (773) 834-7698; E-mail: hdew@midway.uchicago.edu

Received January 29, 2002; revised April 4, 2002; accepted April 9 , 2002.

Online publication: 4/9/02 at www.acnp.org/citations/ Npp040902281.
These behaviors include an inability to inhibit inappropriate behaviors, an inability to wait, an alteration in time perception, or a relative insensitivity to negative consequences, particularly delayed or uncertain negative consequences or rewards (Logan and Cowan 1984; Rachlin and Green 1972; Ainslie 1975; Meck 1996; Mischel et al. 1989; Barkley 1997). These behavioral patterns may contribute to the inappropriate use of drugs, insofar as they impair the ability to refrain from engaging in potentially damaging behaviors. In addition, these behavioral tendencies may also emerge as a consequence of using drugs, either in the short term (i.e., after acute administration) or after chronic drug use. That is, ingestion of a drug may lead to an impaired ability to wait or evaluate consequences, and this, in turn, may have ad- 
verse consequences. We have a limited understanding of the relationship between each of these behavioral indices of impulsivity and drug use, either as a determinant or a consequence of drug use. The present study is part of a series of studies investigating the relationships among different forms of impulsivity, and the effects of drugs of abuse on these behaviors.

In the present study we studied the effects of an acute dose of $d$-amphetamine on several indices of impulsivity in healthy adults. There were two opposing hypotheses. $d$-Amphetamine is known to have a high potential for abuse, and drugs of abuse are commonly believed to increase maladaptive and risky behaviors. However, $d$-amphetamine is also effective in the treatment of children and adults with Attention Deficit Hyperactivity Disorder (ADHD; American Psychiatric Association (APA) 1994; Solanto 1998; Spencer et al. 2001). In these populations it reduces many of the symptoms of impulsivity, inattention and restlessness. Thus, viewing $d$-amphetamine as a drug of abuse, we expected that it would increase impulsivity, but viewing it as a medication we expected that it would decrease impulsivity.

Impulsivity was measured in this study using five behavioral tests, each based on different operational definitions of impulsivity. Each of the tests has been previously used as an index of impulsivity, albeit with different subject populations and addressing different experimental questions. One goal of the present study was to investigate whether $d$-amphetamine differentially affects performance on different measures of impulsivity. The first behavioral measure used was the delay discounting task, which assesses preference for more immediate and more certain rewards, relative to delayed or uncertain rewards (Rachlin and Green 1972; Logue 1988). More impulsive individuals prefer more immediate rewards (Petry 2001a,b; Crean et al. 2000). The second procedure was the Stop Task, which assesses the ability to inhibit a prepotent response (Logan et al. 1984). Children with ADHD, who are highly impulsive, are impaired on the Stop Task. The third task was the delay of gratification procedure, which measures the ability to withhold a response for a small reward for a period of time, in order to obtain a larger reward. This type of procedure was originally used with children (Mischel et al. 1989), but it has been adapted for use with adults (Newman et al. 1992). The fourth task was the Go/No-Go procedure, which measures complex decision-making including the withholding of inappropriate choices. The fifth task was a measure of time perception. Barkley (1997) has argued that impulsivity in children with ADHD is related to impairment in the perception of time reflected in an overestimation of elapsed time in a test of time reproduction. Although each of these five measures has been used in at least one clinical context as an index of impulsive behavior, their relationships to one another and their relative sensitiv- ity to interventions believed to affect impulsivity are not known.

The main goal of this project was to investigate the effects of a single dose of a stimulant drug, $d$-amphetamine, on these behavioral measures of impulsivity in healthy human volunteers. $d$-Amphetamine is a prototypic stimulant which increases synaptic levels of dopamine and noradrenaline (Koob and Bloom 1988; Kuczenski and Segal 1997). Although it is a drug of abuse and can produce euphoria and feelings of wellbeing in humans, this drug also has beneficial effects on behavior in patients with ADHD. Consistent with its therapeutic effects, Richards and colleagues reported that acute administration of a stimulant drug decreased impulsive behavior in rats, as measured by a delay discounting procedure, and that a dopamine antagonist increased delay discounting, i.e., made the rats more impulsive (Wade et al. 2000; Richards et al. 1999a). Cardinal et al. (2000) examined the effects of $d$-amphetamine on delay discounting using a slightly different procedure in which they compared the drug's effects in the presence or absence of a cue during the delay. In their study $d$-amphetamine decreased preference for a large reinforcer relative to a smaller, more immediate reinforcer when no cue was present, but it increased preference for the larger reinforcer when the cue was present. This suggests that the effects of dopaminergic drugs on impulsivity depend on the presence or absence of conditioned cues. Other investigators (e.g., Logue et al. 1992; Evenden and Ryan 1996; Charrier and Thiebot 1996) have found that acute doses of stimulant drugs increase impulsivity on delay discounting procedures. The procedural differences that account for these varying results across the studies have yet to be identified.

The effects of $d$-amphetamine have also been examined using the Stop Task. Children with ADHD are impaired in the ability to inhibit a prepotent response, and this impairment is reversed with methylphenidate (Tannock et al. 1989). Although the effects of $d$-amphetamine on the Stop Task have not been examined in healthy control children, other studies suggest that $d$ amphetamine produces similar effects in healthy children as it does in children with ADHD (Rapoport et al. 1978). Fillmore and Rush (2002) recently reported that cocaine users are impaired in their ability to inhibit responses, compared with non-cocaine-using controls. However, it is not possible to determine whether these differences already existed or resulted from the cocaine use. In healthy adult volunteers (de Wit et al. 2000), we found that acute doses of $d$-amphetamine improved the ability to inhibit responses only in individuals who were initially relatively poor at inhibiting their responses at baseline. This can be seen as parallel to the findings with children with ADHD, who were also relatively poor in inhibiting responses before the drug. Feola et al. (2000) found that $d$-amphet- 
amine also improved the ability to inhibit responses in rats at doses that did not affect simple response reaction times. Thus, $d$-amphetamine appears to improve the ability to inhibit responses, but its effects may depend on baseline levels of impulsivity.

Other studies have examined the effects of $d$-amphetamine on the perception of time, and found that stimulants result in the subjective perception that time is passing more rapidly. This phenomenon has been interpreted as indicative of an increase in the speed of the internal "clock" (Meck 1996). This finding would lead us to expect that in our study subjects will underestimate the intervals in the time estimation tasks.

The goal of this study was to evaluate the effects of moderate doses of $d$-amphetamine (10 and $20 \mathrm{mg}$ ) on five behavioral measures of impulsive behavior in healthy volunteers. Measures of the subjective effects of $d$-amphetamine were also taken to confirm that the drug was producing its predicted subjective effects. Based on previous studies of the effects of stimulant drugs on measures of impulsive behavior it was hypothesized that acute doses of $d$-amphetamine would decrease impulsivity. However, the relationships among the different measures of impulsivity are not known, and it is not known whether they reflect a single, or multiple, underlying processes. Thus, it was possible that $d$-amphetamine would differentially affect performance on the different measures. The responses of male and female subjects were compared, and in exploratory analyses subjects' responses were also examined in relation to circulating levels of cortisol and gonadal hormones.

\section{METHODS}

\section{Subjects}

Thirty-six healthy volunteers (18 male, 18 female), aged 18 to 45 , participated in the study. Volunteers were recruited from the university and surrounding community via posters, advertisements in newspapers, and word-of-mouth referrals. Initial eligibility was ascertained during a telephone interview, and appropriate candidates were scheduled for a face-to-face interview. Candidates completed a psychiatric symptom checklist (SCL-90; Derogatis 1983), the Michigan Alcoholism Screening Test (Selzer 1971), and a health questionnaire that included a detailed section on current and lifetime recreational drug use and history. A semi-structured psychiatric interview was conducted to rule out participants who met criteria for major DSM IV diagnoses (APA 1994). All subjects received a physical examination and an electrocardiogram. Exclusion criteria included any history of an Axis I psychiatric disorder (including substance use disorders), any significant medical problems, an abnormal electrocardiogram, a body mass index outside the $19-26 \mathrm{~kg} / \mathrm{m}^{2}$ range, less than a high school education, smoking more than five cigarettes per day, absence of past recreational drug use, and lack of English fluency. The University of Chicago Hospital Institutional Review Board approved the protocol. The consent form stated that the purpose of the study was to test the effects of drugs on mood and behavior. For blinding purposes the consent form listed drugs in addition to those that were administered in the study, namely stimulant/appetite suppressant, sedative/tranquilizer, cannabinoid/marijuana-like drug, alcohol, and placebo. Subjects agreed to abstain from drugs, other than their usual amounts of caffeine and nicotine, for $24 \mathrm{~h}$ before and $6 \mathrm{~h}$ after each session. Subjects were paid for their participation.

\section{Design}

The study utilized a double-blind, placebo-controlled, within-subject design. Each subject participated in three experimental sessions during which they received capsules containing either $d$-amphetamine (10 or $20 \mathrm{mg}$ ) or placebo. Drug condition orders were randomized and sessions were scheduled allowing $72 \mathrm{~h}$ for drug clearance. Subjects were tested individually in comfortably furnished rooms containing sofas, tables, a television with $\mathrm{VCR}$, a radio, and magazines. When dependent measures were not being collected, subjects were allowed to relax and watch television or movies, play games, or read, but were not allowed to work or study. Subjects were transported home at the end of each session.

\section{Session Protocol}

Subjects participated in three 4-h sessions conducted from 9:00 A.M. to 1:00 P.M. Upon arrival at the laboratory, urine was tested for recent drug use and pregnancy, and a breath alcohol level (BAL) was obtained. No BAL readings or drug screens were positive. At 09:10 A.M., subjects completed baseline subjective effects questionnaires (see below), performed nonspecific tests of psychomotor function (Digit Symbol Substitution Test; DSST; Wechsler 1958) and memory (the Hopkins Verbal Learning Test and Digit Span (Wechsler 1958)), and provided a pre-drug saliva sample to measure levels of cortisol and testosterone for exploratory analyses. Ovarian hormones were also tested, but because of excessive variability will not be reported here. Vital signs were also recorded at this time. At 10:10 A.M., the subjects ingested two capsules containing either $d$-amphetamine (10 or $20 \mathrm{mg}$ ) or placebo with 100 $\mathrm{ml}$ water. The subjective effects questionnaires, DSST, and vital signs were re-administered at 10:35, 11:00, and 
11:25 A.M. Ninety minutes after ingesting the capsule (11:40 A.M.), subjects began to perform the five behavioral tasks designed to measure impulsivity (see below). All subjects completed the tasks in the same order: Go/No-Go, Delay Discounting, Stop Task, Delay of Gratification, and Time Estimation. These tasks took approximately $60 \mathrm{~min}$ to complete. After subjects finished the tasks (12:40 P.M.), a final set of subjective effects questionnaires, memory tests, and the DSST were administered, vital signs were recorded, and a second saliva sample was collected (post-drug). Subjects completed an end-of-session questionnaire and were transported home at 1:00 P.M. After they completed the last session, a debriefing interview was conducted during which participants completed personality questionnaires (not reported here) and received payment.

\section{Drugs}

d-Amphetamine (Dexedrine; Smith Kline Beecham, Philadelphia, PA; $5 \mathrm{mg}$ ) tablets were administered in opaque, colored gelatin capsules (size 00) with dextrose filler. The doses of 10 and $20 \mathrm{mg} d$-amphetamine are known to produce modest but reliable subjective and behavioral effects in healthy volunteers (Brauer and de Wit 1996). Placebo capsules contained only dextrose.

\section{Dependent Measures}

Vital Signs. Blood pressure and heart rate were assessed using a Digital Blood Pressure Monitor HEM706 (Omron Healthcare, Vernon Hills, IL).

Addiction Research Center Inventory (ARCI: Martin et al. 1971). The 49-item ARCI is a true-false questionnaire with five empirically derived scales that are sensitive to the effects of several classes of abused drugs. The five scales measure drug-induced euphoria (MorphineBenzedrine Group; MBG), stimulant-like effects (Amphetamine; A), intellectual efficiency and energy (Benzedrine Group; BG), sedation (Pentobarbital-Chlorpromazine; PCAG), and dysphoria (Lysergic Acid; LSD). The ARCI has been widely used in studies of abuse liability, and is considered to be valid and reliable (Fischman and Foltin 1991; Martin et al. 1971).

Drug Effects Questionnaire (DEQ; Johanson and Uhlenhuth 1980). The DEQ consists of four questions concerning current drug effects. Subjects indicate on a 100$\mathrm{mm}$ line (from "not at all" to "very much") whether they are feeling any drug effects, if they like them, if they are high, and if they want more of the drug.

Profile of Mood States (POMS; McNair et al. 1971; Johanson and Uhlenhuth 1980). This version of the POMS consists of 72 adjectives commonly used to describe momentary mood states. Subjects rate from 0 (not at all) to 5 (extremely) the extent to which each adjective describes how they feel at that moment. The items on the POMS have been factor analyzed to yield eight mood state scales: Anger, Anxiety, Confusion, Depression, Elation, Fatigue, Friendliness, and Vigor. In addition, the POMS has two intuitively derived scales: Arousal ((Anxiety + Vigor) - (Fatigue + Confusion)) and Positive Mood (Elation - Depression).

\section{General Measures of Performance}

Digit Symbol Substitution Test (DSST; Wechsler 1958). The DSST is a pencil and paper test of psychomotor and cognitive performance in which subjects are required to substitute a series of numbers for symbols within $90 \mathrm{~s}$. The number of correct responses in $90 \mathrm{~s}$ is recorded. Five equivalent forms are used to minimize order and learning effects.

The Hopkins Verbal Learning Test (HVLT; Brandt 1991). The HVLT is a test of verbal recall consisting of three trials of immediate free recall followed by a delayed free recall trial and a delayed recognition trial. A list of twelve words from four groups of semantically related words is read to the subjects. Immediately following the reading of the list, subjects are asked to recall as many words as possible. This procedure is repeated three times. Twenty-five minutes later, subjects are asked to recall the words from the list, and then to perform a recognition task in which they must identify words from the original list from among both semantically related and unrelated words. Six versions of the HVLT were used to minimize learning.

Digit Span (Wechsler 1958). The Digit Span is a memory task involving series of numbers ranging from two to eight digits in length. Subjects are read progressively longer series of digits and then asked to immediately recall them either as read (Digits Forward) or backward (Digits Backward). Different series are used in each section. There are six versions of the Digit Span to reduce learning across trials.

\section{Behavioral Measures of Impulsivity}

Stop Task (Logan et al. 1997). The Stop Task is designed to assess the subject's ability to inhibit a prepotent response. Subjects are instructed to respond as quickly as possible when a specific letter (Go signal) is presented on a computer screen, and to inhibit (Stop) their responses when a tone is presented very soon after the Go signal. The tone is presented on random trials and at different delays following each letter presentation. The delays to the Stop signal are varied systematically according to the subject's performance: the delay to the tone is adjusted until the subject inhibits (Stops) his or her responses on approximately $50 \%$ of trials. After the Stop signal delay has been adjusted to this $50 \%$ 
criterion, the time required for the subject to stop the go response (Stop reaction time; Stop RT) can be determined. The Stop RT is calculated by subtracting the final mean delay at which the tone is presented from the mean Go RT. This is the primary dependent measure of this task. The Go reaction time (Go RT), or latency to respond to the letter presentation, is a secondary dependent measure, a measure of simple reaction time. Both Go RTs and Stop RTs are measured in milliseconds.

Go/No-Go (Newman et al. 1985). This is a test of learning which is designed to assess the ability to inhibit an inappropriate response. The task consists of repeated presentations of eight pairs of numbers, four of which are designated "correct" and four as incorrect. Subjects receive rewards (10 cents) for responding correctly to the correct numbers, and they lose 10 cents for responding to the incorrect numbers. The subject's task is to respond to the correct numbers only, and to withhold responses to the incorrect numbers. The subjects are required to learn which pairs are correct and incorrect by experiencing the consequences. The outcome measures are errors of omission (withholding a response when a correct stimulus is presented), and errors of commission or false alarms (i.e., responding to an incorrect stimulus). Errors of commission were the primary measure of impulsivity in this task. Hits are the number of go responses to which subjects correctly respond. Total Correct refers to the number of hits minus the false alarms. Eighty trials are presented at $2 \mathrm{sec}$ intervals. Different number pair lists were presented on each session to limit learning across sessions.

Delay Discounting (Richards et al. 1997, 1999b). Delay discounting provides an index of the relative value of immediate vs. delayed rewards. This task uses a computerized adjusting amount procedure to measure discounting of delayed and uncertain reinforcers. Subjects have the opportunity to choose between different amounts of money available after varying delays or probabilities. The test consists of about 110 questions, such as: (1) Would you rather have $\$ 10.00$ in 30 days or $\$ 2.00$ at the end of the session, or (2) Would you rather have $\$ 5.00$ for sure or $\$ 10.00$ with a $25 \%$ chance? At the end of the session, when all 110 questions have been answered, one question/response is selected at random and the subject receives whatever they chose in response to that question. If on that trial they selected an immediate amount of money, they receive the money in cash immediately. If they selected delayed money, the money is provided when the time has elapsed. If they selected a probabilistic amount, subjects draw a token from a bag containing two colors of tokens in the proportion that reflects the probability, and the subject receives the amount of money indicated by the color of the token immediately in cash. The questions are presented on a computer screen according to an adjusting amount procedure (Richards et al. 1997, 1999b), in which the amount of immediate, certain money is adjusted across successive questions (trials) until an amount is reached that is judged by the participant to be equivalent to the delayed (delay trials) or uncertain $\$ 10$ reward (probability trials). The amount of immediate, certain money the subject judges to be equivalent to the $\$ 10$ reward thus provides a quantitative measure of the subjective value of the delayed or uncertain rewards. These points of subjective equality are called indifference points. Delay and probability trials are intermixed. Within each session, indifference points are determined for five different delays, $0,2,30,180$, and 365 days, and five different probabilities, 1.0, 0.9, 0.75, 0.5 and 0.25 . On delay trials, participants choose between a varying amount of money available immediately and $\$ 10$ available after a delay. On probability trials, participants choose between a variable amount of money delivered for-sure $(p=1.0)$ and $\$ 10$ to be delivered on a probabilistic basis. The indifference points obtained at each of the delays and probabilities are plotted and discount functions are derived through curve-fitting analyses. This yields a parameter $\mathrm{k}$ : higher values of $\mathrm{k}$ are taken to indicate greater impulsivity. Similar procedures are used to derive the probability discounting function (see Richards et al, 1999b for details).

Delay of Gratification (Newman et al. 1992). This task provides a measure of subjects' willingness to wait for a reward. Subjects may choose to respond by pressing on either of two buttons, one associated with immediate reward (5 cents) with a relatively low probability, the other associated with a delay but also a higher probability of reward. That is, during the task one button is associated with high-density reward (HDR; $80 \%$ payoff) with a 10-s delay in the opportunity to respond, whereas the other button is associated with lower density reward (LDR; $40 \%$ payoff) but the subject is able to respond immediately. Subjects can earn as much as $\$ 2.00$ if they always choose the HDR button, whereas they earn only $\$ 1.00$ if they always choose the LDR button. Thus, their relative preference for the HDR versus the LDR button is considered an index of the subject's acceptance or avoidance of the 10-s delay. Subjects get immediate feedback on their responses. The words "You win" or "You lose" appear on the screen, and a counter on the screen indicates their accumulated earnings. The procedure consists of 50 test trials under each condition. The primary dependent measure is the proportion of trials on which subjects choose the delayed option. Subjects who choose the delayed option less frequently are considered to be more impulsive.

Time Perception. Time perception was measured using a time estimation task (Lange et al. 1995). In the time estimation task, subjects are presented with a picture of two 
light bulbs depicted side by side on a computer screen. A trial begins when the light bulb on the left (display stimulus) illuminates. The duration of the visual stimulus is 2 $\mathrm{s}$ and $4 \mathrm{~s}$ in random order over 10 trials. During illumination of the display stimulus subjects are asked to attend to distractor figures (e.g. cartoon insects) that appear and move across the screen, and are told that they will be asked specific questions concerning the distractor figures at the end of the test. Immediately after the display stimulus extinguishes the subject is instructed to illuminate the bulb on the right for an amount of time as similar to the display stimulus as they can by holding down a key on the keyboard. Accuracy of time estimation is calculated as a percentage relationship between estimated time and actual time.

\section{Data Analysis}

All dependent measures that were administered repeatedly throughout the testing session (e.g. ARCI, Vitals, DSST) were analyzed using 2-way ANOVAs (Drug $\times$ Time). The behavioral tasks were compared using 1-way ANOVAs (Drug). Tukey Honestly Significant Difference (HSD) post hoc comparisons were conducted on all main effects and interactions yielding significant F-values. Sex differences were examined using an additional factor in the ANOVA. The significance level for all statistical tests was set at $p<.05$. Some further exploratory analyses were conducted using Pearson Product Moment correlations.

\section{RESULTS}

\section{Subject Demographics}

The demographic characteristics and drug use histories of the thirty-six participants in the study are summarized in Table 1.

\section{Vitals}

$d$-Amphetamine significantly increased heart rate, systolic BP, and diastolic BP $(d$-amphetamine $\times$ Time interaction). Tukey HSD post hoc comparisons $(p<.05)$ revealed that heart rate and $\mathrm{BP}$ were dose dependently elevated after $d$-amphetamine, relative to placebo, between 75 and 150 min after drug administration, and that the effects of the high dose were greater than the low dose.

\section{Cortisol and Testosterone}

$d$-Amphetamine significantly increased cortisol levels (1-way ANOVA performed on post-drug assays, $\mathrm{F}_{2,70}=$ 13.236, $p<.001$ ). Post hoc comparisons revealed that
Table 1. Subject Demographic and Drug Use Summary $(n=36)$

\begin{tabular}{|c|c|}
\hline \multicolumn{2}{|l|}{ Age (years) } \\
\hline Range & $18-44$ \\
\hline Mean \pm SD & $24 \pm 6.5$ \\
\hline Weight (lbs: mean \pm SD) & $145.8 \pm 21.6$ \\
\hline \multicolumn{2}{|l|}{$\operatorname{Sex}(\mathrm{n})$} \\
\hline Male & 18 \\
\hline Female & 18 \\
\hline \multicolumn{2}{|l|}{ Race/Ethnicity (n) } \\
\hline Caucasian & 23 \\
\hline African-American & 1 \\
\hline Asian & 6 \\
\hline Hispanic & 6 \\
\hline \multicolumn{2}{|l|}{ Education (n) } \\
\hline Partial college & 22 \\
\hline College degree & 11 \\
\hline Advanced degree & 3 \\
\hline Full time student & 22 \\
\hline \multicolumn{2}{|l|}{ Current drug use } \\
\hline Alcohol (mean \pm SD; drinks/week) & $6.5 \pm 4.8$ \\
\hline Caffeine (mean \pm SD; drinks/week) & $8.4 \pm 8.2$ \\
\hline Cigarettes ( $\mathrm{n}:>2.5$ cigarettes / day) & 9 \\
\hline Marijuana (n: >0.5 cigarettes/week) & 2 \\
\hline \multicolumn{2}{|l|}{ Lifetime drug use } \\
\hline Stimulants (n: ever used) & 10 \\
\hline Tranquilizers (n: ever used) & 4 \\
\hline Hallucinogens (n: ever used) & 16 \\
\hline Opiates (n: ever used) & 3 \\
\hline \multicolumn{2}{|l|}{ Marijuana } \\
\hline Never used (n) & 7 \\
\hline Used < 10 times $(\mathrm{n})$ & 6 \\
\hline Used 10-50 times (n) & 12 \\
\hline Used $>50$ times $(\mathrm{n})$ & 11 \\
\hline Inhalants (n: ever used) & 8 \\
\hline
\end{tabular}

both $d$-amphetamine doses increased cortisol relative to placebo, and the high dose produced a greater effect than the low dose (mean \pm SEM cortisol levels for placebo $=0.20 \pm 0.02,10 \mathrm{mg}=0.28 \pm 0.02$, and $20 \mathrm{mg}=$ $0.35 \pm 0.03)$. Baseline (pre-capsule) testosterone levels were higher in men than in women (mean \pm SEM $=$ $113.92 \pm 10.42$ and $52.66 \pm 8.90 \mathrm{pg} / \mathrm{ml}$, respectively) but were not altered by $d$-amphetamine and were not related to task performance.

\section{Subjective Effects}

$d$-Amphetamine increased subjects' ratings on the ARCI A, BG, LSD, and MBG scales (significant $d$-amphetamine $\times$ Time interaction; Table 2). On the ARCI A scale, post hoc comparisons revealed that the low dose $(10 \mathrm{mg})$ increased scores relative to placebo after 75 and $150 \mathrm{~min}$, while the high dose $(20 \mathrm{mg})$ increased scores relative to both placebo and the low dose at 50, 75, and $150 \mathrm{~min}$. The high dose also significantly increased scores, compared with placebo and the low dose, on the ARCI BG and MBG scales at 50, 75, and $150 \mathrm{~min}$. The low dose increased scores on the MBG scale at 50 and $75 \mathrm{~min}$. 
Table 2. Significant F Values (ANOVA) for Main Effects of $d$-Amphetamine and Interactions of $d$-Amphetamine and Time within Session (Time)

\begin{tabular}{lcc}
\hline Dependent Measure & $d$-Amphetamine & $d$-Amphetamine $\times$ Time \\
\hline Vital Signs & & $6.07^{* * *}$ \\
Heart Rate & $6.14^{* * *}$ & $7.98^{* * *}$ \\
Systolic BP & & $4.15^{* * *}$ \\
Diastolic BP & $13.24^{* * *}$ & $-\mathrm{NA}-$ \\
Salivary Cortisol & & \\
DEQ & $26.46^{* * *}$ & $8.17^{* * *}$ \\
Feel & $3.97^{*}$ & $2.12^{*}$ \\
Like & $12.53^{* * *}$ & $5.65^{* * *}$ \\
High & $3.57^{*}$ & $2.71^{* *}$ \\
Want More & & $9.31^{* * *}$ \\
ARCI & $14.47^{* * *}$ & $3.93^{* * *}$ \\
A & $10.24^{* * *}$ & $4.61^{* * *}$ \\
BG & $3.25^{*}$ & $7.55^{* * *}$ \\
LSD & $7.73^{* * *}$ & $2.37^{*}$ \\
MBG & & $5.68^{* * *}$ \\
POMS & & $4.37^{* * *}$ \\
Friendliness & $5.67^{* *}$ & $3.52^{* * *}$ \\
Anxiety & & \\
Vigor & & \\
Arousal & $3.19^{*}$ & \\
DSST & & \\
Digit Span & & \\
\hline$* p<.05, * * p .01, * * p<.001$ & &
\end{tabular}

d-Amphetamine dose-dependently increased scores on the POMS Elation, Vigor, Friendliness, and Arousal scales (significant Drug $\times$ Time interactions; Table 2). At the lower dose these effects occurred only at $75 \mathrm{~min}$, whereas at the higher dose scores were elevated from $50 \mathrm{~min}$ to $150 \mathrm{~min}$ (Figure 1).

$d$-Amphetamine dose-dependently increased DEQ ratings of "feel drug," "like drug," "want more," and "feel high" (significant Drug $\times$ Time interaction; Table 2) at 75 and $150 \mathrm{~min}$.

\section{General Psychomotor and Memory Effects}

$d$-Amphetamine improved performance on the DSST (Drug $\times$ Time interaction; Table 2, Figure 1) and digit span test (main effect of drug). On the DSST, post hoc comparisons revealed that both $d$-amphetamine doses significantly increased the number of symbols correctly completed on the DSST after 75 and $150 \mathrm{~min}$. Digit span performance increased with dose. Verbal recall, as measured by the Hopkins Verbal Learning Test, was not affected by the drug.

\section{Behavioral Task Performance}

On the Stop Task, $d$-amphetamine significantly reduced Stop RT $\left(\mathrm{F}_{2,66}=3.205, p<.05\right.$; Figure 2). Post hoc comparisons indicated that the reduction in Stop RT was significant relative to placebo after the high dose only (mean $=164 \mathrm{~ms}$ compared with $193 \mathrm{~ms}$ ). d-Amphetamine did not affect Go RT at either dose, indicating that the drug affected response inhibition specifically, not just overall reaction time (Figure 2). Because we had found in a previous study (de Wit et al. 2000) that $d$-amphetamine decreased Stop RT only in subjects who exhibited slow Stop RTs on the placebo session, a similar analysis was conducted here. Slow and fast Stop RT groups were created by median split of placebo session performance and the groups were then compared on their response to $d$-amphetamine on Stop and Go RT. This analysis revealed that $d$-amphetamine specifically reduced Stop RT in the slow stoppers, and not in subjects with initially fast Stop RTs (Figure 3).

On the Go/No-Go task, both doses of $d$-amphetamine decreased the number of errors of commission (i.e., false alarms), relative to placebo $\left(\mathrm{F}_{2,66}=3.416, p<\right.$ .05; Figure 3), but did not affect the errors of omission. Thus, the effects of $d$-amphetamine were specific to errors of commission. $d$-Amphetamine also decreased reaction time at the lower dose $\left(\mathrm{F}_{2,66}=3.216, p<.05\right.$; Figure 4). Both doses of $d$-amphetamine increased the total number of correct responses on the task (hits - false alarms), relative to placebo $\left(\mathrm{F}_{2,66}=3.416, p<.05\right)$. To examine individual differences on the effects of the drug (as described above and in de Wit et al. 2000), two groups were created by median split of number of false 


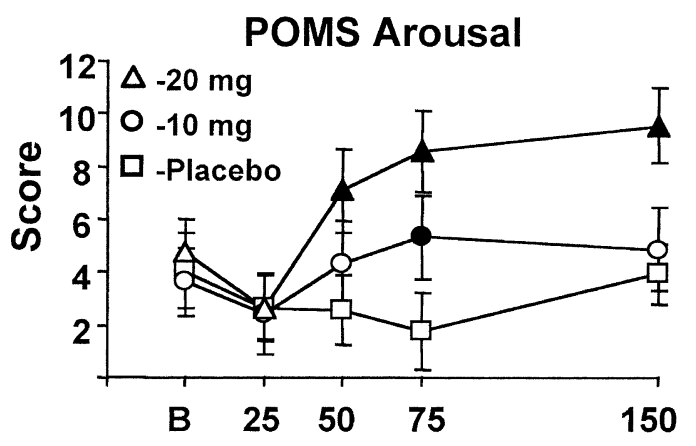

Time ( $\min )$
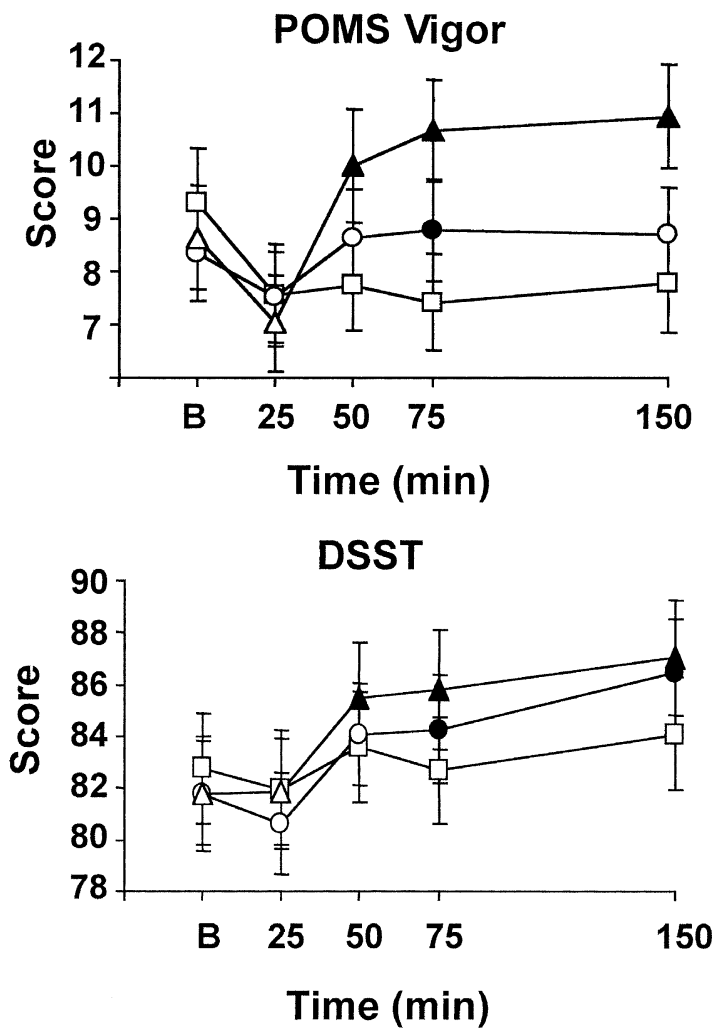

Figure 1. Mean ( \pm SEM) scores for POMS Arousal, POMS Vigor, and DSST (number of symbols correctly completed) at baseline (B), 25, 50, 75, and $150 \mathrm{~min}$ after capsule. Filled symbols on POMS graphs indicate that the mean is significantly different from both $10 \mathrm{mg}$ and placebo (20 mg value; triangles) or from placebo (10 mg values; circles). Filled symbols on the DSST graph indicate that the mean is significantly different from placebo.

alarms made on the placebo session. These groups were compared on task performance after $d$-amphetamine. As in the Stop Task, there were significant differences in the effects of $d$-amphetamine between these two groups of responders (significant Group X Drug interaction). $d$-Amphetamine significantly reduced false alarm rates in the high false alarm group, but did not affect false alarm rates in the low false alarm group.
Stop Task
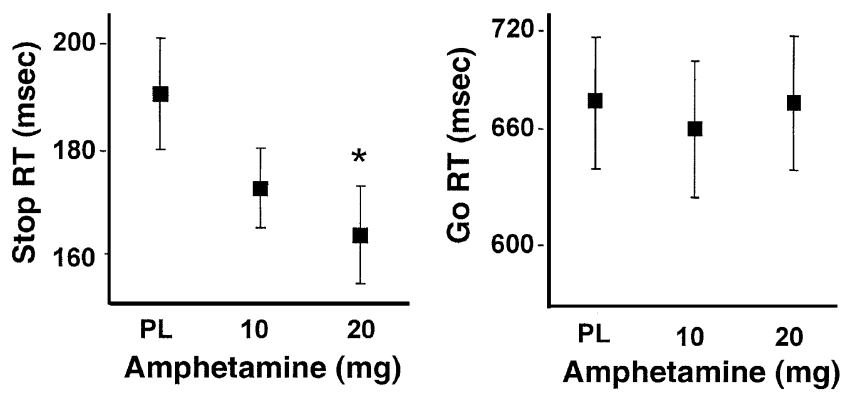

Figure 2. Mean ( \pm SEM) Stop Task Stop RT and Go RT (msec) after placebo (PL), 10, and $20 \mathrm{mg} d$-amphetamine. *Significant difference between placebo Stop RT and $20 \mathrm{mg}$.

On the discounting task we found that the hyperbolic discount function was a good fit for both the delay and the probability indifference points, resulting in average $\mathrm{r}^{2}$ values of .87 for delay and .90 for probability discounting. $d$-Amphetamine significantly reduced the $\mathrm{k}$ values on the delay discounting task (i.e., decreased delay discounting) after the high $d$-amphetamine dose only (mean \pm SEM $\mathrm{k}$ for placebo $=.037 \pm .01$ and mean $\mathrm{k}$ value after $20 \mathrm{mg} d$-amphetamine $=.025 \pm .009$ ). On the probability Discounting task there was a non-significant trend for a decrease in discounting of the less probable rewards $\left(\mathrm{F}_{2,64}=2.638, p=.079\right)$. The effects of $d$-amphetamine were not different in subjects who exhibited high vs. low discounting on the placebo session.

$d$-Amphetamine did not significantly affect performance on the Delay of Gratification test. d-Amphetamine did not alter the number of HDR button responses, the primary measure $\left(\mathrm{F}_{2,68}=1.817, p>.05\right.$; mean \pm SEM placebo response $=19.77 \pm 1.49,10 \mathrm{mg}$ $d$-amphetamine $=17.09 \pm 1.69,20 \mathrm{mg}=19.83 \pm 1.34)$,

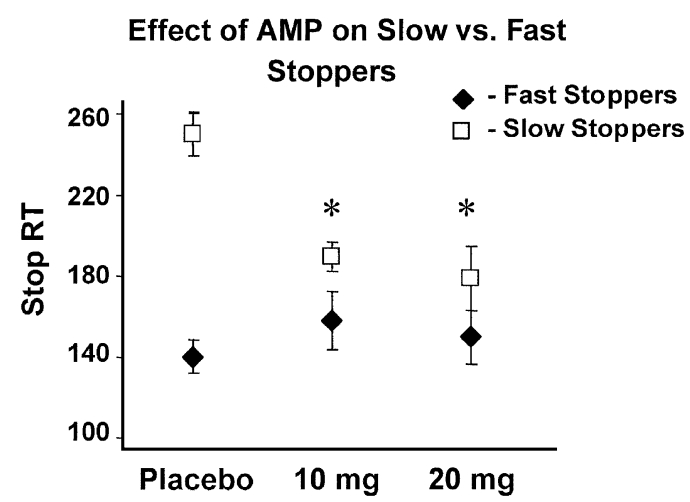

Figure 3. Mean ( \pm SEM) Stop RT (msec) in slow and fast stoppers after placebo, 10, and $20 \mathrm{mg} d$-amphetamine. *Stop RT after 10 and $20 \mathrm{mg}$ in slow stoppers is significantly different from placebo. 

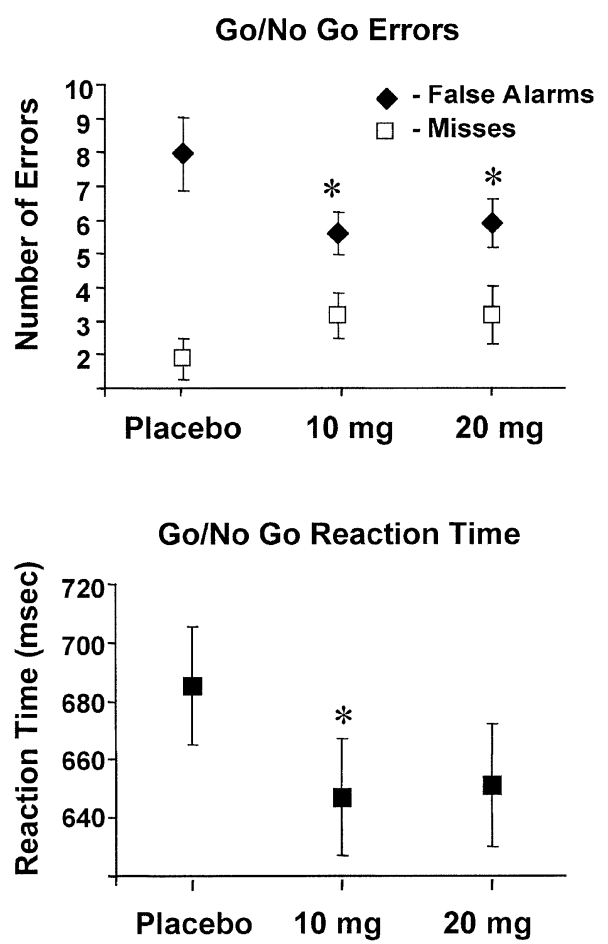

Figure 4. Mean ( \pm SEM) number of false alarms and misses on the Go/No-Go task and Go/No-Go reaction time (msec). *Significant differences from placebo after $10 \mathrm{mg}$ and $20 \mathrm{mg}$ on false alarms, and after $10 \mathrm{mg}$ on reaction time.

nor did it affect a second measure, total earnings $\left(\mathrm{F}_{2,68}=\right.$ 2.023, $p>.05$ ).

Finally, $d$-amphetamine produced a non-significant trend in time perception. Both doses of $d$-amphetamine reduced subjects' estimates of time relative to actual time. The length of time that subjects estimated was converted to a percentage of the actual duration, and averaged over the two time intervals ( $2 \mathrm{~s}$ and $4 \mathrm{~s}$ ) yielding a total performance score. After placebo, subjects' mean estimates of actual time were about $93 \%$ (mean \pm SEM estimate for 2 -s interval $=1.97 \pm 0.12 \mathrm{~s} ; 4$-s inter$\mathrm{val}=3.50 \pm 0.17 \mathrm{~s}$ ), and after $d$-amphetamine these decreased to $88 \%$ (mean \pm SEM estimate for 2-s interval = $1.82 \pm 0.12 \mathrm{~s} ; 4$-s interval $=3.41 \pm 0.18 \mathrm{~s}$ ) and $89 \%$ (mean \pm SEM estimate for 2-s interval $=1.83 \pm 0.12 \mathrm{~s}$; 4 -s interval $=3.45 \pm 0.19 \mathrm{~s}$ ) after 10 and $20 \mathrm{mg}$ respectively. Although these effects were not significant they are in the direction of a quickened perception of time after $d$-amphetamine. The effects of $d$-amphetamine were not different in subjects who exhibited high vs. low estimates of time after placebo.

\section{Post-hoc Analyses}

Several exploratory analyses were conduced to characterize the results more completely. First, we conducted an analysis of sex differences, and found no significant differences between men and women. We also analyzed the relation between performance on the impulsivity tasks and the euphorigenic effects of $d$-amphetamine. Two groups were formed based on subjects' responses on the ARCI MBG scale, a measure of euphoria, after $20 \mathrm{mg} d$-amphetamine: subjects who experienced the greatest increase in MBG scores vs. subjects who experienced the least increase. These two groups were compared on measures of impulsivity on the placebo session (baseline), and after $d$-amphetamine (i.e., change from placebo to $d$-amphetamine). Two interesting relations emerged: (1) subjects who reported the least increase in euphoria after $d$-amphetamine were most impulsive on the Go/No-Go task at baseline; and (2) $d$-amphetamine decreased the false alarm rate among the subjects who experienced low euphoria after the drug, whereas it did not affect false alarm rate in subjects who reported significant euphoria after $d$-amphetamine (Figure 5). This suggests that there may be a relationship between this measure of impulsivity and the euphorigenic effects of drugs. No relationships were found between $d$-amphetamine-induced euphoria and other indices of impulsivity. Finally, we examined relationships among the five behavioral tasks during the placebo session. A modest positive correlation was obtained between Time Estimation and Delay of Gratification (percent of actual duration and HDR button responses; $r=.366, p=.03$ ). However, in view of the number of correlations conducted in this analysis (10) this finding must be interpreted with caution.

\section{DISCUSSION}

In the present study, low-to-moderate doses of $d$-amphetamine decreased impulsive patterns of responding on

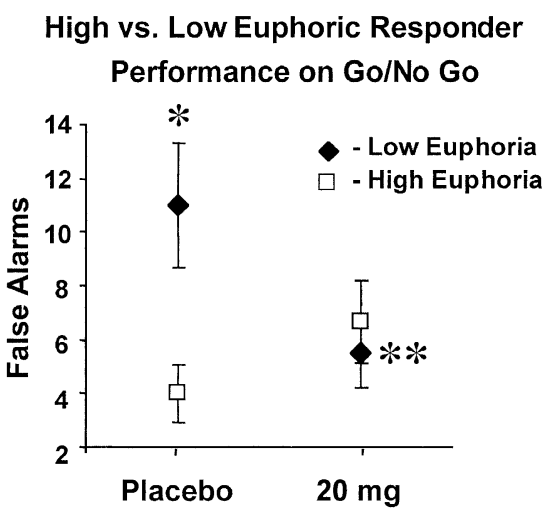

Figure 5. Mean ( \pm SEM) false alarms on the Go/No-Go task in subjects with a high and low euphoria response to 20 mg $d$-amphetamine. *Low and high euphoria subjects significantly differ in number of false alarms at baseline (placebo). **False alarm rate in the low euphoria group is significantly reduced after $20 \mathrm{mg} d$-amphetamine. 
three measures of impulsive behavior, two of which assess behavioral inhibition and one of which measures the relative value of immediate vs. delayed rewards. $d$-Amphetamine significantly decreased the time it took subjects to inhibit a prepotent response on the Stop Task (i.e., decreased Stop RT), and it reduced the number of false alarms on the Go/No-Go task. On both of these tasks the effects of $d$-amphetamine were specific, insofar as the drug did not affect other measures not considered indicative of impulsive responding (e.g., Go reaction time in the Stop Task or misses on the Go/NoGo task). $d$-Amphetamine also made subjects perform less impulsively on the delay discounting task. On this task, $d$-amphetamine decreased the $\mathrm{k}$ value, indicating less steep discounting of delayed rewards. It is notable that the drug's effects were consistent on these very different measures of impulsive behavior: two measures of inhibition and a cognitive measure of choice and decision making. $d$-Amphetamine did not significantly affect performance on the delay of gratification task or the time estimation task.

The results on the Stop Task replicate and extend previous studies on the effects of $d$-amphetamine on impulsivity. For example, we previously reported that $d$-amphetamine $(20 \mathrm{mg}$ ) decreased Stop RT only in subjects who exhibited relatively long Stop RTs without drug administration (de Wit et al. 2000). Similar results were obtained in the present study. These effects of $d$-amphetamine on inhibition are consistent with those observed in children with ADHD (Tannock et al. 1989). Without drug treatment, children with ADHD exhibit longer Stop RTs than control children, but this deficit is reversed during treatment with methylphenidate, a $d$-amphetamine-like drug which improves their clinical symptoms. The improvement of Stop RT is also consistent with previous studies in rats (Feola et al. 2000). In that study, as well, acute doses of $d$-amphetamine decreased Stop RT, consistent with a decrease in impulsive responding.

The second measure on which $d$-amphetamine improved impulsive responding was the Go/No-Go task, a choice procedure in which subjects are required to withhold a response on certain trials in order to obtain rewards. $d$-Amphetamine decreased the rate of false alarms, a measure of the ability to inhibit inappropriate responses. This outcome may be viewed as similar to the improved Stop RT. The Stop Task measures the time it takes to inhibit an ongoing response, while the Go/No-Go procedure measures the proportion of responses that are successfully inhibited. Thus, these two measures of impulsive behavior share some underlying processes. It is also notable that on both the Stop Task and the Go/No-Go task the effects of $d$-amphetamine were most marked in subjects whose initial level of performance was relatively poor (i.e., subjects with initially slow Stop RTs and subjects with initially high rates of false alarms), further suggesting commonalities between the measures. It will be of interest to determine whether these two measures covary in other situations, such as across subjects who vary in the trait of impulsivity, or in response to other drugs and treatments that are thought to affect this form of impulsivity.

The third measure of impulsivity that was altered by $d$-amphetamine was the delay discounting procedure, a measure that is conceptually quite different from the Stop Task and the Go/No-Go task. Whereas the Stop Task and Go/No-Go tasks are measures of the ability to inhibit response, delay discounting reflects a more cognitive process involving the evaluation of delayed vs. immediate outcomes. Delayed rewards are generally valued less than more immediate rewards, and this tendency is especially pronounced among impulsive individuals (e.g., Crean et al. 2000; Petry 2001a,b). Stimulant drugs decrease delay discounting in rats (i.e., decrease impulsive responding; Richards et al. 1999a), but this has not been previously demonstrated in humans. Indeed, this is the first demonstration that acute administration of any drug can alter delay discounting in humans. It is notable that the direction of the effect of $d$-amphetamine was consistent with that observed in laboratory animals, and it is also consistent with the decreases in impulsivity observed with the other measures of impulsivity in the present study. These findings lend support to the validity of the delay discounting procedure as a measure of impulsive behavior, and support the general conclusion that acute administration of stimulant drugs decreases impulsive behavior.

$d$-Amphetamine did not change performance on the delay of gratification or time estimation tasks. The delay of gratification task has not been widely used with normal adults or in studies involving drug administration. It was developed and is commonly used with children (Mischel et al. 1989), but to our knowledge the only study to have previously used it with adults is Newman et al. (1992), which compared psychopathic subjects to nonpsychopathic controls. Although the psychopathic subjects performed more poorly on the task, their results suggested that the procedure could be used with healthy controls. Without further data about the task, its suitability for non-psychopathic adults, and its relative sensitivity to trait or state variations in impulsivity, it is somewhat difficult to interpret the absence of a drug effect in the present study. $d$-Amphetamine did not significantly alter time estimation, although there was a non-significant trend toward shorter time estimates after $d$-amphetamine. The direction of this effect is consistent with previous reports on the effects of stimulants on time estimation (Meck 1996).

In the present study, $d$-amphetamine also improved a non-specific measure of performance, the DSST, and a measure of memory, digit span. On one hand this might indicate that the drug non-specifically improves 
performance (i.e., on general measures of psychomotor performance as well as those designed to measure impulsivity) perhaps by affecting alertness or attention. Another interpretation of these findings, however, is that the DSST and memory tests also involve components of behavioral inhibition. That is, $d$-amphetamine may improve performance on these more general tasks through its ability to inhibit inappropriate responses.

Although we had no a priori reason to expect that the mood altering effects of $d$-amphetamine would be related to its effects on impulsivity, we conducted an exploratory analysis that yielded interesting results. Subjects were divided into two groups, based on their peak ratings of euphoria after $20 \mathrm{mg} d$-amphetamine, as measured by the ARCI MBG scale (Low Euphoria $n=18$, High Euphoria $n=$ 18). The performance of these two groups on the tasks were compared first on the placebo (non-drug) sessions and it was found that the Low Euphoria group made significantly more false alarms on the Go/No-Go task than the High Euphoria group. Then the performance of the two groups on the tasks was compared after $d$-amphetamine administration. Here, it was found that $d$-amphetamine decreased the rate of false alarms in the Low Euphoria group but not in the High Euphoria group. It is of interest that this relationship does not simply reflect the magnitude of subjects' responses to $d$-amphetamine on all measures: the drug produced greater effects on euphoria in the High Euphoria group, but it produced more pronounced effects on impulsive behavior in the Low Euphoria group. These results suggest that there may be common mechanisms (e.g. dopaminergic) between the effects of $d$-amphetamine on mood and its effects on impulsive behavior. $d$-Amphetamine is an indirect agonist of dopamine and norepinephrine (Kuczenski and Segal 1997). Whereas the abuse potential of $d$-amphetamine is thought to be related to its effects at the dopamine (DA) receptor (Wise and Bozarth 1987), it is less clear if the effects of $d$-amphetamine on impulsive behavior are mediated by DA as well. In support of a role for DA in impulsive behavior we recently reported that D2 antagonists such as flupenthixol and raclopride increased impulsive behavior in rats (Wade et al. 2000). Nevertheless, other neurotransmitter systems affected by $d$-amphetamine, such as norepinephrine, may also contribute to the behavioral changes reported here.

In some ways it seems paradoxical that DA may be involved in the positive reinforcing effects of $d$-amphetamine and also decrease impulsivity. One way to reconcile these apparently contradictory roles is to consider that DA may be involved in the salience and impact of conditioned stimuli. Studies in rats have shown that DA agonists such as $d$-amphetamine increase the value of conditioned reinforcers and that 6-hydroxydopamine lesions of the nucleus accumbens block this effect (Taylor and Robbins 1984, 1986). An increased salience and impact of conditioned stimuli is consistent with the improved responsivity to performance-related stimuli in the Stop Task, and the Go/No-Go task. The increased salience of conditioned stimuli may also contribute to likelihood of $d$-amphetamine or other stimulants to be abused (Phillips and Fibiger 1990).

The finding that $d$-amphetamine decreases impulsive behavior is also not consistent with clinical impressions that chronic drug users are impulsive. It should be noted that the moderate acute doses of $d$-amphetamine that decreased impulsive behavior in the present study are very different from the large chronic doses of $d$-amphetamine consumed by drug abusers. Indeed, it has been suggested that low doses of $d$-amphetamine may affect presynaptic autoreceptors with a net effect opposite to the usual increases in synaptic levels (Solanto 1998). In a recent paper with rats, Richards et al. (1999a) found that while moderate acute doses decreased impulsive behavior, large chronic doses of methamphetamine increased impulsive behavior on the delay discounting task. These differential effects may be related to doses, or duration of exposure to $d$-amphetamine.

In summary, the present study demonstrated that $d$-amphetamine decreased impulsive responding on several behavioral tasks designed to measure different components of impulsivity. These effects were specific to measures of impulsivity, rather than to general measures such as simple reaction time. It is notable that $d$-amphetamine decreased impulsive patterns of responding on two very different measures of impulsive behavior, delay discounting and behavioral inhibition, and that the results on these measures are concordant with preclinical studies. These findings leave little doubt that low, acute doses of stimulant drugs improve performance on these measures. It is important to consider the performance-enhancing effects of $d$-amphetamine together with the abuse potential of the drug. Acute moderate doses of $d$-amphetamine appear to have effects opposite to those of chronic large doses of $d$-amphetamine. In studies with rats we have found that large chronic doses of $d$-amphetamine increase impulsivity, and high doses of $d$-amphetamine taken by human drug abusers may increase impulsivity. An important question then, is at what dose or degree of chronic intake do the effects of $d$-amphetamine shift from decreasing impulsive behavior to increasing impulsivity, and what are the changes in the brain that are responsible for this dramatic shift in behavioral effects. The answers to these questions may be crucial for understanding and solving the problem of drug abuse.

\section{ACKNOWLEDGMENTS}

This research was funded by the National Institute on Drug Abuse (DA09133). The authors thank Laura Schleifer, Ph.D., for her valuable assistance. 


\section{REFERENCES}

Ainslie G (1975): Specious reward: A behavioral theory of impulsiveness and impulse control. Psychol Bull 82: 463-496

American Psychiatric Association (1994): Diagnostic and Statistical Manual of Mental Disorders, 4th Edition. Washington DC, American Psychiatric Association

Barkley RA (1997): ADHD and the Nature of Self-control. New York, The Guildford Press

Brandt J (1991): The Hopkins Verbal Learning Test: Development of a new memory test with six equivalent forms. Clin Neuropsychol 5:125-142

Brauer LH, de Wit H (1996): Subjective responses to $d$-amphetamine alone and after pimozide pretreatment in normal, healthy volunteers. Biol Psychiatry 39:26-32

Cardinal RN, Robbins TW, Everitt BJ (2000): The effects of $d$-amphetamine, chlordiazepoxide, alpha-flupenthixol and behavioral manipulations on choice of signaled and unsignaled delayed reinforcement in rats. Psychopharmacology (Berl) 152:362-375

Charrier D, Thiebot MH (1996): Effects of psychotropic drugs on rat responding in an operant paradigm involving choice between delayed reinforcers. Pharmacol Biochem Behav 54:149-157

Crean JP, de Wit H, Richards JB (2000): Reward discounting as a measure of impulsive behavior in a psychiatric outpatient population. Exp Clin Psychopharmacol 8:155162

de Wit H, Crean J, Richards JB (2000): Effects of $d$-amphetamine and ethanol on a measure of behavioral inhibition in humans. Behav Neurosci 114:830-837

Derogatis L (1983): SCL-90-R Manual-II. Towson, MD, Clinical Psychometric Research

Evenden JL, Ryan CN (1996): The pharmacology of impulsive behaviour in rats: The effects of drugs on response choice with varying delays of reinforcement. Psychopharmacology (Berl) 128:161-170

Feola TW, Richards JB, de Wit H (2000): Effects of $d$-amphetamine and alcohol on a measure of behavioral inhibition in rats. Behav Neurosci 114:838-848

Fillmore MT, Rush CR (2002): Impaired inhibitory control of behavior in chronic cocaine users. Drug and Alcohol Dependence 66:265-273

Fischman MW, Foltin RW (1991): Utility of subjective-effects measurements in assessing abuse liability of drugs in humans. Br J Addict 86:1563-1570

Johanson CE, Uhlenhuth EH (1980): Drug preference and mood in humans: Diazepam. Psychopharmacology (Berl) 71:269-273

Koob GF, Bloom FE (1988): Cellular and molecular mechanisms of drug dependence. Science 242:715-723

Kuczenski R, Segal DS (1997): Effects of methylphenidate on extracellular dopamine, serotonin, and norepinephrine: Comparison with amphetamine. J Neurochem 68:20322037

Lange KW, Tucha O, Steup A, Gsell W, Naumann M (1995): Subjective time estimation in Parkinson's disease. J Neural Transm (Suppl 46):433-438
Logan GD, Cowan WB (1984): On the ability to inhibit thought and action: A theory of an act of control. Psychol Rev 91:295-327

Logan GD, Cowan WB, Davis KA (1984): On the ability to inhibit simple and choice reaction time responses: A model and a method. J Exp Psychol Hum Percept Perform 10:276-291

Logan GD, Schachter RJ, Tannock R (1997): Impulsivity and inhibitory control. Psychol Sci 8:60-64

Logue AW (1988): Research on self-control: An integrated framework. Behav Brain Sci 11:665-709

Logue AW, Tobin H, Chelonis JJ, Wang RY, Geary N, Schachter S (1992): Cocaine decreases self-control in rats: A preliminary report. Psychopharmacology (Berl) 109:245-247

Martin WR, Sloan JW, Sapira JD, Jasinski DR (1971): Physiologic, subjective and behavioral effects of amphetamine, methamphetamine, ephedrine, phenmetrazine, and methylphenidate in man. Clin Pharmacol Ther 12:245258

McNair D, Lorr M, Droppleman L (1971): Profile of Mood States. San Diego, Educational and Industrial Testing Service

Meck WH (1996): Neuropharmacology of timing and time perception. Brain Res Cogn Brain Res 3:227-242

Mischel W, Shoda Y, Rodriguez ML (1989): Delay of gratification in children. Science 244:933-938

Newman JP, Kosson DS, Patterson CM (1992): Delay of gratification in psychopathic and nonpsychopathic offenders. J Abnorm Psychol 101:630-636

Newman JP, Widom CS, Nathan S (1985): Passive avoidance in syndromes of disinhibition: Psychopathy and extraversion. J Person Soc Psychol 48:1316-1327

Petry NM (2001a): Delay discounting of money and alcohol in actively using alcoholics, currently abstinent alcoholics, and controls. Psychopharmacology (Berl) 154:243250

Petry NM (2001b): Pathological gamblers, with and without substance use disorders, discount delayed rewards at high rates. J Abnorm Psychol 110:482-487

Phillips AG, Fibiger HC (1990): Role of reward and enhancement of conditioned reward in persistence of responding for cocaine. Behav Pharmacol 1:269-282

Rachlin H, Green L (1972): Commitment, choice, and selfcontrol. J Exp Anal Behav 17:15-22

Rapoport JL, Buchsbaum MS, Zahn TP, Weingartner H, Ludlow C, Mikkelsen EJ (1978): Dextroamphetamine: cognitive and behavioral effects in normal prepubertal boys. Science 199:560-563

Richards JB, Mitchell SH, de Wit H, Seiden LS (1997): Determination of discount functions in rats with an adjusting amount procedure. J Exp Anal Behav 67:353-366

Richards JB, Sabol KE, de Wit H (1999a): Effects of methamphetamine on a model of impulsive behavior in rats. Psychopharmacology (Berl) 146:432-439

Richards JB, Zhang L, Mitchell SH, de Wit H (1999b): Delay or probability discounting in a model of impulsive behavior: Effect of alcohol. J Exp Anal Behav 71:121-143

Selzer ML (1971): The Michigan Alcoholism Screening Test: 
The quest for a new diagnostic instrument. Am J Psychiatry 127:1653-1658

Solanto MV (1998): Neuropsychopharmacological mechanisms of stimulant drug action in attention-deficit hyperactivity disorder: a review and integration. Behav Brain Res 94:127-152

Spencer T, Biederman J, Wilens T, Faraone S, Prince J, Gerard K, Doyle R, Parekh A, Kagan J, Bearman SK (2001): Efficacy of a mixed amphetamine salts compound in adults with attention-deficit/hyperactivity disorder. Arch Gen Psychiatry 58:775-782

Tannock R, Schachar RJ, Carr RP, Chajczyk D, Logan GD (1989): Effects of methylphenidate on inhibitory control in hyperactive children. J Abnorm Clin Psychol 17:473491

Taylor JR, Robbins TW (1984): Enhanced behavioral control by conditioned reinforcers following microinjections of $d$-amphetamine into the nucleus accumbens. Psychopharmacology (Berl) 84:405-412

Taylor JR, Robbins TW (1986): 6-Hydroxydopamine lesions of the nucleus accumbens, but not the caudate-nucleus, attenuate enhanced responding with reward-related stimuli produced by intra-accumbens $d$-amphetamine. Psychopharmacology (Berl) 90:390-397

Wade TR, de Wit H, Richards JB (2000): Effects of dopaminergic drugs on delayed reward as a measure of impulsive behavior in rats. Psychopharmacology (Berl) 150: 90-101

Wechsler D (1958): The Measure and Appraisal of Adult Intelligence. Baltimore, Williams and Wilkins

Wise RA, Bozarth MA (1987): A psychomotor stimulant theory of addiction. Psychol Rev 94:469-492 